\author{
M. Akhmet ${ }^{1 \text { iD }}$, N. Aviltay ${ }^{2 *}$, Zh. Artykbayeva ${ }^{2}$ \\ ${ }^{1}$ Middle East Technical University, Ankara, Turkey \\ ${ }^{2}$ Faculty of Mechanical-mathematics, al-Farabi Kazakh national university, Almaty, Kazakhstan \\ *e-mail: avyltay.nauryzbay@gmail.com
}

\title{
ASYMPTOTIC BEHAVIOR OF THE SOLUTION OF THE INTEGRAL BOUNDARY VALUE PROBLEM FOR SINGULARLY PERTURBED INTEGRO-DIFFERENTIAL EQUATIONS
}

The work is devoted to clarifying asymptotic with respect to a small parameter behavior of the solution of the integral boundary value problem for singularly perturbed linear integro-differential equation. We study the boundary value problem for singularly perturbed integro-differential equations with the phenomena of the so-called boundary jumps, when the fast solution variable becomes unbounded at both boundaries. The exceptions of the qualitative influence of integral terms on the asymptotic behavior of the solutions for singularly perturbed integro-differential equations are shown. The presence of integral terms will significantly change the degenerate equation: the solution of the assumed singularly perturbed integro-differential equation does not tend to the solution of the usual degenerate equation, obtained from the supposed equation with the zero value of a small parameter and will tend to solve a specially modified degenerate integrodifferential equation with an additional term called the jump of the integral term. Boundary and initial functions are defined; their existence and uniqueness are proved. On the basis of the constructed boundary and initial functions are obtained analytical formula and asymptotic estimates of the solution for the integral boundary value problem. It is established that the solution of the considered boundary value problem at the ends of a given segment has the phenomena of boundary jumps of the same orders. A modified degenerate boundary value problem is constructed, to the solution of which approaches the solution of assumed singularly perturbed integral boundary value problem. The value of the jump of integral terms is found. An example was made based on the initial results.

Key words: singular perturbation, small parameter, the initial jump, asymptotics.

$$
\begin{gathered}
\text { M. Ахмет }{ }^{1}, \text { Н. Авилтай } \\
\end{gathered}
$$

Сингулярлы ауытқыған интегралды-дифференциалдық теңдеуге арналған шекаралық есеп шешімінің асимптотикалық сипаты

Жұмыс сингулярлы ауытқыған интегро-дифференциалдық теңдеу үшін шекаралық есеп шешімінің кіші параметр бойынша асимптотикалық құбылысын анықтауға арналған. Біз сингулярлы ауытқыған интегро-дифференциалдық теңдеуді, кіші параметр нөлге ұмтылғанда шешім кесіндінің екі жақ шетінде шексіздікке ұмтылатын шекаралық секіріс құбылысымен қарастырамыз. Жұмыста интегралдық мүшенің сингулярлы ауытқыған интегро-дифференциалдық теңдеу үшін шекаралық есеп шешімінің асимптотикалық сипатына әсері көрсетілген. Интегралдық мүше ауытқымаған шекаралық есепті айтарлықтай өзгертеді. Кіші параметр нөлге ұмтылған кезде сингулярлы ауытқыған шекаралық есеп шешімі қарапайым ауытқымаған шекаралық есеп шешіміне ұмтылмайды. Сингулярлық ауытқыған шекаралық есеп шешімі интегралдық мүшенің секірісі бар өзгертілген ауытқымаған есеп шешіміне ұмтылады. 
Asymptotic behavior of the solution ...

Жұмыста интегралдық шекаралық есеп шешімінің аналитикалық формуласы мен асимптотикалық бағалаулары алынды. Берілген кесіндінің екі жақ шетінде де қарастырылып отырған шекаралық есеп шешімінің бастапқы секіріс құбылысының реті бірдей екендігі анықталды. Осы сингулярлы ауытқыған шекаралық есебіне сәйкес өзгертілген ауытқымаған шекаралық есеп құрылған. Берілген сингулярлы ауытқыған шекаралық есеп шешімінің өзгертілген ауытқымаған шекаралық есебіне ұмтылатыны дәлелденген. Интегралдық мүшенің бастапқы секірісінің шамасы анықталды. Мысал бастапқы нәтижелер негізінде жасалды.

Түйін сөздер: сингулярлық ауытқу, кіші параметр, бастапқы секіріс, асимптотика.

\author{
М. Ахмет $^{1}$, Н. Авилтай ${ }^{2}$, Ж. Артыкбаева ${ }^{2}$ \\ ${ }^{1}$ Средне-восточный технический университет, Анкара, Турция \\ ${ }^{2}$ Казахский национальный университет им. Аль-Фараби, Алматы, Казахстан \\ *e-mail: avyltay.nauryzbay@gmail.com
}

\title{
Асимптотическое поведение решения интегральной краевых задач для сингулярно возмущенных интегро-дифференциальных уравнений
}

Работа посвящена выяснению асимптотического по малому параметру поведения решения интегральной краевой задачи для сингулярного возмущенного линейного интегродифференциального уравнения. Мы изучаем краевую задачу для сингулярно возмущенных интегро-дифференциальных уравнений с явлениями так называемых граничных скачков, когда переменная быстрого решения становится неограниченной на обеих границах. Показаны исключения качественного влияния интегральных членов на асимптотическое поведение решений сингулярно возмущенных интегро-дифференциальных уравнений. Наличие интегральных членов существенно изменит вырожденное уравнение. Решение предполагаемого сингулярно возмущенного интегро-дифференциального уравнение не стремится к решению обычного вырожденного уравнения, полученного из предполагаемого уравнения с нулевым значением малого параметра, и будет стремиться специально модифицированное вырожденное интегро-дифференциальное уравнение с дополнительным членом, называемым скачком интегрального члена. В работе получены аналитическая формула и асимптотические оценки решения интегральной краевой задачи. Установлено, что решение рассматриваемой краевой задачи на концах заданного отрезка имеет явления граничных скачков тех же порядков. Построена модифицированная вырожденная краевая задача, к решению которой стремится решение исходной сингулярно возмущенной интегральной краевой задачи. Определена величина начального скачка интегрального члена. Пример был сделан на основе начальных результатов.

Ключевые слова: малый параметр, сингулярное возмущение, асимптотика, начальный скачок.

\section{Introduction}

Singularly perturbed equations is called equations that contain a small parameter with higher derivatives. Many applied problems in physics, mechanics, technology, etc. are modeled using this type of equations in mathematics. In the following work of the authors L. Schlesinger [1], G.D. Birkhoff [2], P. Noaillon [3], W. Wasow [4], A.H. Nayfeh [5], A. N. Tikhonov [6,7], M.I. Vishik, L.A. Lusternik [8,9], N.N. Bogolyubov, U.A Mitropolsky [10], A.B. Vasilieva and V.F. Butuzov [11,12], R.E. O’Malley [13,14], D.R. Smith [15], W. Eckhaus [16], K. W. Chang and F. A. Howes [17], J. Kevorkian and J.D. Cole [18], Sanders and F. Verhulst [19], E.F. Mischenko, N. Rozov [20], S.A. Lomov [21], M.I. Imanaliev [22], K.A.Kassymov [23-25] was appeared and devoloped the theory of such type equations.

In the study of certain singularly perturbed problems, it can be found that the fast variable of the solution near the boundary of the set takes on an infinitely large value, since 
the small parameter tends to zero. The study of the Cauchy problem with an initial jump for a single nonlinear ODE was began by authors' work L.A. Lusternik and M.I. Vishik [26] and K.A.Kassymov [27]. The most common cases of the Cauchy problem with initial jump were studied by K.A. Kassymov. A characteristic exception of such problems is that the solution of this problem approaches to solution of degenerate equation with modified initial conditions when the small parameter goes to zero. In this instance, it is said that the phenomenon of the initial jump for the solution is valid.

In [28-31] was investigated boundary value problems (BVP) for singularly perturbed ODE and integro-differential equations with initial jumps.

In $[32,33]$ was studied BVP for integro-differential equations third-order with a small parameter at two higher derivatives, when hold the so-called phenomena of boundary jumps, i.e. when some derivatives of the solution for sufficiently small values of the parameter become infinitely large at both ends of the interval. But as this takes place at the ends of the considered interval of solving these problems, there were jumps of different orders. Singularly perturbed differential equations with a piecewise constant argument considered in [34].

In the present work, we investigate integral BVP for singularly perturbed linear integrodifferential equations third-order, solution that at the ends of a given segment has jumps of the same order. The main goal of this paper is to establish the asymptotic behavior of the solution on a small parameter and the construction of the modified degenerate problem. A similar work but without integral conditions considered in [35].

\section{Statement of the problem and auxiliary materials}

Consider the following singularly perturbed linear integro-differential equation

$$
L_{\mu} y \equiv \mu^{2} y^{\prime \prime \prime}+\mu A_{0}(t) y^{\prime \prime}+A_{1}(t) y^{\prime}+A_{2}(t) y=F(t)+\int_{0}^{1} \sum_{i=0}^{2} H_{i}(t, x) y^{(i)}(x, \mu) d x
$$

with integral boundary conditions

$$
h_{1} y \equiv y(0, \mu)=\alpha, \quad h_{2} y \equiv y^{\prime}(0, \mu)=\beta, \quad h_{3} y \equiv y^{\prime}(1, \mu)-\int_{0}^{1} \sum_{i=0}^{2} a_{i}(x) y^{(i)}(x, \mu) d x=\gamma
$$

where $\mu>0$ is a small parameter, $\alpha, \beta, \gamma$ are known constants independent of $\mu$.

Assume that following conditions hold:

C1) Functions $A_{i}(t), i=\overline{0,2}, F(t), a_{i}(t), i=\overline{0,2}$ are defined on the interval $0 \leq t \leq$ 1, $H_{0}(t, x), H_{1}(t, x), H_{2}(t, x)$ are defined in the domain $D=\{0 \leq t \leq 1,0 \leq x \leq 1\}$ and sufficiently smooth.

C2) The roots $\kappa_{i}(t), i=1,2$ of "additional characteristic equation" $\kappa^{2}+A_{0}(t) \kappa+A_{1}(t)=0$ satisfy the following inequalities $\kappa_{1}(t) \leqslant-\gamma_{1}<0, \kappa_{2}(t) \geqslant \gamma_{2}>0$.

C3) $a_{2}(1) \neq 1$.

We consider homogeneous singularly perturbed equation associated with (1):

$$
L_{\varepsilon} y \equiv \mu^{2} y^{\prime \prime \prime}+\mu A_{0}(t) y^{\prime \prime}+A_{1}(t) y^{\prime}+A_{2}(t) y=0 \text {. }
$$


The fundamental set of solutions of equation (3) takes the form [35]

$$
\begin{aligned}
& y_{1}^{(q)}(t, \mu)=\frac{1}{\mu^{q}} e^{\frac{1}{\mu} \int_{0}^{t} \kappa_{1}(x) d x} \cdot\left(\kappa_{1}^{q}(t) y_{10}(t)+O(\mu)\right), \quad q=\overline{0,2}, \\
& y_{2}^{(q)}(t, \mu)=\frac{1}{\mu^{q}} e^{-\frac{1}{\mu} \int_{t}^{1} \kappa_{2}(x) d x} \cdot\left(\kappa_{2}^{q}(t) y_{20}(t)+O(\mu)\right), \quad q=\overline{0,2} \\
& y_{3}^{(q)}(t, \mu)=y_{30}^{(q)}(t)+O(\mu), \quad q=\overline{0,2},
\end{aligned}
$$

where $y_{30}(t)=e^{-\int_{0}^{t} \frac{A_{2}(x)}{A_{1}(x)} d x}$, functions $y_{i 0}(t), i=1,2$ are solutions of the problem:

$$
p_{i}(t) \cdot y_{i 0}^{\prime}(t)+q_{i}(t) \cdot y_{i 0}(t)=0, \quad y_{i 0}(0)=1, \quad i=1,2,
$$

where

$$
p_{i}(t)=\left(A_{0}(t)+2 \kappa_{i}(t)\right) \kappa_{i}(t), \quad q_{i}(t)=3 \kappa_{i}(t) \kappa_{i}^{\prime}(t)+A_{0}(t) \kappa_{i}^{\prime}(t)+A_{2}(t) .
$$

Wronskian, composed of the fundamental system of solutions of equation (3) has the form:

$$
\begin{array}{r}
W(t, \mu)=\frac{1}{\mu^{3}} e^{\frac{1}{\mu} \int_{0}^{t} \kappa_{1}(x) d x-\frac{1}{\mu} \int_{t}^{1} \kappa_{2}(x) d x}\left(y_{10}(t) y_{20}(t) y_{30}(t) \kappa_{1}(t) \kappa_{2}(t) .\right. \\
\left.\cdot\left(\kappa_{2}(t)-\kappa_{1}(t)\right)+O(\mu)\right) .
\end{array}
$$

We introduce the functions [35]

$$
K_{0}(t, s, \mu)=\frac{P_{0}(t, s, \mu)}{W(s, \mu)} ; \quad K_{1}(t, s, \mu)=\frac{P_{1}(t, s, \mu)}{W(s, \mu)},
$$

where $P_{0}(t, s, \mu), P_{1}(t, s, \mu)$ are the third order determinant derived from the Wronskian $W(s, \mu)$ by replacing the third row with $y_{1}(t, \mu), 0, y_{3}(t, \mu)$ and $0, y_{2}(t, \mu), 0$ respectively. Sum of $K_{0}(t, s, \mu)$ and $K_{1}(t, s, \mu)$ are the Cauchy function. Therefore, these functions have the following properties:

1. With respect to the variable $t$ satisfy equation (3), i.e.

$$
L_{\mu} K_{0}(t, s, \mu)=0, \quad L_{\mu} K_{1}(t, s, \mu)=0, \quad t \in[0,1], \quad t \neq s .
$$

2. When $t=s$ satisfy the conditions:

$$
K_{0}(s, s, \mu)+K_{1}(s, s, \mu)=0, \quad K_{0}^{\prime}(s, s, \mu)+K_{1}^{\prime}(s, s, \mu)=0, \quad K_{0}^{\prime \prime}(s, s, \mu)+K_{1}^{\prime \prime}(s, s, \mu)=1 .
$$

For the functions $K_{0}(t, s, \mu), K_{1}(t, s, \mu)$ in view (4), (5), (6) the following asymptotic representation hold as $\mu \rightarrow 0$ [35]:

$$
\begin{aligned}
K_{0}^{(q)}(t, s, \mu)=\mu^{2} & \left(\frac{y_{30}^{(q)}(t)}{y_{30}(s) \kappa_{1}(s) \kappa_{2}(s)}-\frac{\kappa_{1}^{q}(t) y_{10}(t)}{\mu^{q} y_{10}(s) \kappa_{1}(s)\left(\kappa_{2}(s)-\kappa_{1}(s)\right)} e^{\frac{1}{\mu} \int_{s}^{t} \kappa_{1}(x) d x}+\right. \\
& +O(\mu)), \quad t \geq s, \quad q=\overline{0,2},
\end{aligned}
$$




$$
K_{1}^{(q)}(t, s, \mu)=\mu^{2}\left(\frac{\kappa_{2}^{q}(t) y_{20}(t)}{\mu^{q} y_{20}(s) \kappa_{2}(s)\left(\kappa_{2}(s)-\kappa_{1}(s)\right)} e^{-\frac{1}{\mu} \int_{t}^{s} \kappa_{2}(x) d x}+O(\mu)\right), t \leq s, q=\overline{0,2} .
$$

Let functions $\Phi_{i}(t, \mu), i=1,2,3$ are solutions for the following problem:

$$
L_{\mu} \Phi_{i}(t, \mu)=0, \quad h_{k} \Phi_{i}(t, \mu)=\delta_{k i}, \quad i, k=1,2,3
$$

where $\delta_{k i}$ is Kronecker symbol. Functions $\Phi_{i}(t, \mu), i=1,2,3$ are called boundary functions. The boundary functions are determined by the formula

$$
\Phi_{i}(t, \mu)=\frac{J_{i}(t, \mu)}{J(\mu)},
$$

where $J(\mu)$ is the determinant consisting of the fundamental solution system of equation (3):

$$
J(\mu)=\left|\begin{array}{lll}
h_{1} y_{1}(t, \mu) & h_{1} y_{2}(t, \mu) & h_{1} y_{3}(t, \mu) \\
h_{2} y_{1}(t, \mu) & h_{2} y_{2}(t, \mu) & h_{2} y_{3}(t, \mu) \\
h_{3} y_{1}(t, \mu) & h_{3} y_{2}(t, \mu) & h_{3} y_{3}(t, \mu)
\end{array}\right|
$$

$J_{i}(t, \mu)$ is the determinant derived from $J(\mu)$ by replacing the $i$-th row by the fundamental set of solutions $y_{1}(t, \mu), y_{2}(t, \mu), y_{3}(t, \mu)$ of the equation (3). From (9), by virtue of (2), (3), we obtain for $J(\mu)$ the asymptotic representation:

$$
J(\mu)=\frac{1}{\mu^{2}}\left(\kappa_{2}(1) y_{20}(1) \kappa_{1}(0)\left(1-a_{2}(1)\right)+O(\mu)\right) \neq 0 .
$$

Then from (8) in view (4), (10) we get asymptotic representation as $\mu \rightarrow 0$ for boundary functions $\Phi_{i}(t, \mu), i=1,2,3$ :

$$
\begin{aligned}
& \Phi_{1}^{(q)}(t, \mu)=y_{30}^{(q)}(t)-\frac{y_{30}^{\prime}(0) \kappa_{1}^{q}(t) y_{10}(t)}{\mu^{q-1} \kappa_{1}(0)} e^{\frac{1}{\mu} \int_{0}^{t} \kappa_{1}(x) d x}- \\
& -\frac{\kappa_{2}^{q}(t) y_{20}(t)\left(h_{3} y_{30}(t)-a_{2}(0) y_{30}^{\prime}(0)\right)}{\mu^{q-1} \kappa_{2}(1) y_{20}(1)\left(1-a_{2}(1)\right)} e^{-\frac{1}{\mu} \int_{t}^{1} \kappa_{2}(x) d x}+ \\
& +O\left(\mu+\frac{1}{\mu^{q-2}} e^{-\frac{t}{\mu} \gamma_{1}}+\frac{1}{\mu^{q-2}} e^{-\frac{1-t}{\mu} \gamma_{2}}\right), q=\overline{0,2}, \\
& \Phi_{2}^{(q)}(t, \mu)=-\mu \frac{y_{30}^{(q)}(t)}{\kappa_{1}(0)}+\frac{\kappa_{1}^{q}(t) y_{10}(t)}{\mu^{q-1} \kappa_{1}(0)} e^{\frac{1}{\mu} \int_{0}^{t} \kappa_{1}(x) d x}-\frac{\kappa_{2}^{q}(t) y_{20}(t) a_{2}(0)}{\mu^{q-1} \kappa_{2}(1) y_{2}(1)\left(1-a_{2}(1)\right)} \\
& \cdot e^{-\frac{1}{\mu} \int_{t}^{1} \kappa_{2}(x) d x}+O\left(\mu^{2}+\frac{1}{\mu^{q-2}} e^{-\frac{t}{\mu} \gamma_{1}}+\frac{1}{\mu^{q-2}} e^{-\frac{1-t}{\mu} \gamma_{2}}\right), q=\overline{0,2} \\
& \Phi_{3}^{(q)}(t, \mu)=\frac{\kappa_{2}^{q}(t) y_{20}(t)}{\mu^{q-1} \kappa_{2}(1) y_{20}(1)\left(1-a_{2}(1)\right)} e^{-\frac{1}{\mu} \int_{t}^{1} \kappa_{2}(x) d x}+O\left(\frac{1}{\mu^{q-2}} e^{-\frac{1-t}{\mu} \gamma_{2}}\right) .
\end{aligned}
$$




\section{Main results}

We are looking for a solution of the boundary value problem $(1),(2)$ in the form:

$$
\begin{aligned}
y(t, \mu)= & C_{1} \Phi_{1}(t, \mu)+C_{2} \Phi_{2}(t, \mu)+C_{3} \Phi_{3}(t, \mu)+ \\
& +\frac{1}{\mu^{2}} \int_{0}^{t} K_{0}(t, s, \mu) v(s, \mu) d s+\frac{1}{\mu^{2}} \int_{1}^{t} K_{1}(t, s, \mu) v(s, \mu) d s,
\end{aligned}
$$

where $\Phi_{i}(t, \mu), i=1,2,3$ are boundary functions and expressed by the formula (8), $K_{0}(t, s, \mu), K_{1}(t, s, \mu)$ are auxiliary functions, determined by the formula (6) and having an asymptotic representation (7), $C_{i}, i=1,2,3$ are unknown constants, $v(t, \mu)$ is an unknown function.

Replacing (12) into equation (1) we get that $v(t, \mu)$ satisfies the following Fredholm integral equation of the second kind:

$$
v(t, \mu)=p(t, \mu)+\int_{0}^{1} H(t, s, \mu) v(s, \mu) d s,
$$

where

$$
\begin{aligned}
& p(t, \mu)=F(t)+C_{1} \int_{0}^{1} \sum_{i=0}^{2} H_{i}(t, x) \Phi_{1}^{(i)}(x, \mu) d x+C_{2} \int_{0}^{1} \sum_{i=0}^{2} H_{i}(t, x) \Phi_{2}^{(i)}(x, \mu) d x+ \\
& +C_{3} \int_{0}^{1} \sum_{i=0}^{2} H_{i}(t, x) \Phi_{3}^{(i)}(x, \mu) d x, \\
& H(t, s, \mu)=\frac{1}{\mu^{2}} \int_{s}^{1} \sum_{i=0}^{2} H_{i}(t, x) K_{0}^{(i)}(x, s, \mu) d x-\frac{1}{\mu^{2}} \int_{0}^{s} \sum_{i=0}^{2} H_{i}(t, x) K_{1}^{(i)}(x, s, \mu) d x .
\end{aligned}
$$

C4) 1 is not an eigenvalue of the kernel $H(t, s, \mu)$.

Taking into account the condition (C4) integral equation (13) has an unique solution, that can be represented in the form

$$
v(t, \mu)=p(t, \mu)+\int_{0}^{1} R_{\mu}(t, s, 1) p(s, \mu) d s,
$$

where $R_{\mu}(t, s, 1)$ is a resolvent of the kernel $H(t, s, \mu)$, representable by (14) as an asymptotic formula $R_{\mu}(t, s, 1)=R_{0}(t, s, 1)+O(\mu)$. Substituting (15) taking account of (14) into (12), we obtain solution of the boundary value problem (1),(2) in the form:

$$
y(t, \mu)=\sum_{i=1}^{3} C_{i} T_{i}(t, \mu)+Q(t, \mu)
$$


where

$$
\begin{aligned}
& T_{i}(t, \mu)=\Phi_{i}(t, \mu)+\frac{1}{\mu^{2}} \int_{0}^{t} K_{0}(t, s, \mu) \overline{\phi_{i}}(s, \mu) d s+\frac{1}{\mu^{2}} \int_{1}^{t} K_{1}(t, s, \mu) \overline{\phi_{i}}(s, \mu) d s, \\
& Q(t, \mu)=\frac{1}{\mu^{2}} \int_{0}^{t} K_{0}(t, s, \mu) \bar{f}(s, \mu) d s+\frac{1}{\mu^{2}} \int_{1}^{t} K_{1}(t, s, \mu) \bar{f}(s, \mu) d s, \\
& \bar{\phi}_{i}(s, \mu)=\int_{0}^{1} \sum_{j=0}^{2} \bar{H}_{j}(s, x, \mu) \Phi_{i}^{(j)}(x, \mu) d x, \bar{f}(s, \mu)=F(s)+\int_{0}^{1} R_{\mu}(s, p, 1) F(p) d p, \\
& \bar{H}_{j}(s, x, \mu)=H_{j}(s, x)+\int_{0}^{1} R_{\mu}(s, p, 1) H_{j}(p, x) d p .
\end{aligned}
$$

For the functions $\bar{H}_{j}(s, x, \mu), \bar{f}(s, \mu)$ are valid asymptotic representations:

$$
\begin{aligned}
& \bar{H} j(s, x, \mu)=H j(s, x)+\int_{0}^{1} R_{0}(s, p, 1) H_{j}(p, x) d p+O(\mu) \equiv \bar{H}_{j}(s, x)+O(\mu), \\
& \bar{f}(s, \mu)=F(s)+\int_{0}^{1} R_{0}(s, p, 1) F(p) d p+O(\mu) \equiv \bar{f}(s)+O(\mu) .
\end{aligned}
$$

For the functions $\bar{\phi}_{i}(s, \mu)$ applying $(11),(18)$, we can obtain the asymptotic formula:

$$
\bar{\phi}_{i}(s, \mu)=\bar{\phi}_{i}(s)+O(\mu), \quad i=\overline{1,3},
$$

where

$$
\begin{aligned}
& \bar{\phi}_{1}(s)=\int_{0}^{1} \sum_{j=0}^{2} \bar{H}_{j}(s, x) y_{30}^{(j)}(x) d x+\bar{H}_{2}(s, 0) y_{30}^{\prime}(0)-\frac{\left(h_{3} y_{30}(t)-a_{2}(0) y_{30}^{\prime}(0)\right)}{1-a_{2}(1)} \bar{H}_{2}(s, 1), \\
& \bar{\phi}_{2}(s)=-\bar{H}_{2}(s, 0)-\bar{H}_{2}(s, 1) \frac{a_{2}(0)}{1-a_{2}(1)}, \quad \bar{\phi}_{3}(s)=\bar{H}_{2}(s, 1) \frac{1}{1-a_{2}(1)} .
\end{aligned}
$$

From (17) applying (7),(11), (18)-(20), for the functions $T_{i}(t, \mu), i=1,2,3, Q(t, \mu)$, we get asymptotic representations:

$$
\begin{gathered}
T_{1}^{(q)}(t, \mu)=y_{30}^{(q)}(t)+\int_{0}^{t} \frac{y_{30}^{(q)}(t) \bar{\phi}_{1}(s)}{y_{30}(s) A_{1}(s)} d s-\frac{y_{10}(t) \kappa_{1}^{q}(t)}{\mu^{q-1} \kappa_{1}(0)}\left(y_{30}^{\prime}(0)+\frac{\bar{\phi}_{1}(0)}{\kappa_{1}(0)\left(\kappa_{2}(0)-\kappa_{1}(0)\right)}\right) . \\
\cdot e^{\frac{1}{\mu} \int_{0}^{t} \kappa_{1}(x) d x}-\frac{y_{20}(t) \kappa_{2}^{q}(t)}{\mu^{q-1} y_{20}(1) \kappa_{2}(1)}\left(\frac{h_{3} y_{30}(t)-a_{2}(0) y_{30}^{\prime}(0)}{1-a_{2}(1)}-\frac{\bar{\phi}_{1}(1)}{\kappa_{2}(1)\left(\kappa_{2}(1)-\kappa_{1}(1)\right)}\right) .
\end{gathered}
$$




$$
\begin{aligned}
& \cdot e^{\frac{1}{\mu} \int_{1}^{t} \kappa_{2}(x) d x}+\frac{\kappa_{1}^{q-2}(t)-\kappa_{2}^{q-2}(t)}{\mu^{q-1}\left(\kappa_{2}(t)-\kappa_{1}(t)\right)} \cdot \bar{\phi}_{1}(t)+ \\
& +O\left(\mu+\frac{1}{\mu^{q-2}} e^{\frac{1}{\mu} \int_{0}^{t} \kappa_{1}(x) d x}+\frac{1}{\mu^{q-2}} e^{\frac{1}{\mu} \int_{1}^{t} \kappa_{2}(x) d x}\right) \\
& T_{2}^{(q)}(t, \mu)=\int_{0}^{t} \frac{y_{30}^{(q)}(t) \bar{\phi}_{2}(s)}{y_{30}(s) A_{1}(s)} d s+\frac{\kappa_{1}^{q-2}(t)-\kappa_{2}^{q-2}(t)}{\mu^{q-1}\left(\kappa_{2}(t)-\kappa_{1}(t)\right)} \cdot \bar{\phi}_{2}(t)- \\
& -\frac{y_{10}(t) \kappa_{1}^{q}(t)}{\mu^{q-1} \kappa_{1}(0)}\left(\frac{\bar{\phi}_{2}(0)}{\kappa_{1}(0)\left(\kappa_{2}(0)-\kappa_{1}(0)\right)}-1\right) e^{\frac{1}{\mu} \int_{0}^{t} \kappa_{1}(x) d x}+ \\
& +\frac{y_{20}(t) \kappa_{2}^{q}(t)}{\mu^{q-1} y_{20}(1) \kappa_{2}(1)}\left(\frac{\bar{\phi}_{2}(1)}{\kappa_{2}(1)\left(\kappa_{2}(1)-\kappa_{1}(1)\right)}-\frac{a_{2}(0)}{1-a_{2}(1)}\right) e^{\frac{1}{\mu} \int_{1}^{t} \kappa_{2}(x) d x}+ \\
& +O\left(\mu+\frac{1}{\mu^{q-2}} e^{\frac{1}{\mu} \int_{0}^{t} \kappa_{1}(x) d x}+\frac{1}{\mu^{q-2}} e^{\frac{1}{\mu} \int_{1}^{t} \kappa_{2}(x) d x}\right) ; \\
& T_{3}^{(q)}(t, \mu)=\int_{0}^{t} \frac{y_{30}^{(q)}(t) \bar{\phi}_{3}(s)}{y_{30}(s) A_{1}(s)} d s-\frac{\kappa_{1}^{q}(t) y_{10}(t) \bar{\phi}_{3}(0)}{\mu^{q-1} \kappa_{1}^{2}(0)\left(\kappa_{2}(0)-\kappa_{1}(0)\right)} e^{\frac{1}{\mu} \int_{0}^{t} \kappa_{1}(x) d x}+ \\
& +\frac{y_{20}(t) \kappa_{2}^{q}(t)}{\mu^{q-1} y_{20}(1) \kappa_{2}(1)}\left(\frac{\bar{\phi}_{3}(1)}{\kappa_{2}(1)\left(\kappa_{2}(1)-\kappa_{1}(1)\right)}+\frac{1}{1-a_{2}(1)}\right) e^{\frac{1}{\mu} \int_{1}^{t} \kappa_{2}(x) d x}+ \\
& +\frac{\kappa_{1}^{q-2}(t)-\kappa_{2}^{q-2}(t)}{\mu^{q-1}\left(\kappa_{2}(t)-\kappa_{1}(t)\right)} \cdot \bar{\phi}_{3}(t)+O\left(\mu+\frac{1}{\mu^{q-2}} e^{\frac{1}{\mu} \int_{0}^{t} \kappa_{1}(x) d x}+\frac{1}{\mu^{q-2}} e^{\frac{1}{\mu} \int_{1}^{t} \kappa_{2}(x) d x}\right) ; \\
& Q^{(q)}(t, \mu)=\int_{0}^{t} \frac{y_{30}^{(q)}(t) \bar{f}(s)}{y_{30}(s) A_{1}(s)} d s-\frac{\kappa_{1}^{q}(t) y_{10}(t) \bar{f}(0)}{\mu^{q-1} \kappa_{1}^{2}(0)\left(\kappa_{2}(0)-\kappa_{1}(0)\right)} e^{\frac{1}{\mu} \int_{0}^{t} \kappa_{1}(x) d x}+ \\
& +\frac{\kappa_{2}^{q}(t) y_{20}(t) \bar{f}(1)}{\mu^{q-1} \kappa_{2}^{2}(1) y_{20}(1)\left(\kappa_{2}(1)-\kappa_{1}(1)\right)} e^{\frac{1}{\mu} \int_{1}^{t} \kappa_{2}(x) d x}+\frac{\kappa_{1}^{q-2}(t)-\kappa_{2}^{q-2}(t)}{\mu^{q-1}\left(\kappa_{2}(t)-\kappa_{1}(t)\right)} \cdot \bar{f}(t)+ \\
& +O\left(\mu+\frac{1}{\mu^{q-2}} e^{\frac{1}{\mu} \int_{0}^{t} \kappa_{1}(x) d x}+\frac{1}{\mu^{q-2}} e^{\frac{1}{\mu} \int_{1}^{t} \kappa_{2}(x) d x}\right) .
\end{aligned}
$$

To find the unknown constants $C_{i}, i=1,2,3$ from (16), taking account of the boundary conditions (2), we get a system of algebraic equations:

$$
\left\{\begin{array}{l}
C_{1} h_{1} T_{1}(t, \mu)+C_{2} h_{1} T_{2}(t, \mu)+C_{3} h_{1} T_{3}(t, \mu)=\alpha-h_{1} Q(t, \mu), \\
C_{1} h_{2} T_{1}(t, \mu)+C_{2} h_{2} T_{2}(t, \mu)+C_{3} h_{2} T_{3}(t, \mu)=\beta-h_{2} Q(t, \mu) . \\
C_{1} h_{3} T_{1}(t, \mu)+C_{2} h_{3} T_{2}(t, \mu)+C_{3} h_{3} T_{3}(t, \mu)=\gamma-h_{3} Q(t, \mu)
\end{array}\right.
$$


For the elements of this system are valid asymptotic representations:

$$
\begin{gathered}
h_{1} T_{1}(t, \mu)=1+O(\mu), h_{1} T_{i}(t, \mu)=O(\mu), i=2,3, \quad h_{1} Q(t, \mu)=O(\mu) \\
h_{2} T_{i}(t, \mu)=-\frac{\bar{\phi}_{i}(0)}{\kappa_{2}(0)\left(\kappa_{2}(0)-\kappa_{1}(0)\right)}+O(\mu), \quad i=1,3, \\
h_{2} T_{2}(t, \mu)=1-\frac{\bar{\phi}_{2}(0)}{\kappa_{2}(0)\left(\kappa_{2}(0)-\kappa_{1}(0)\right)}+O(\mu), \\
h_{2} Q(t, \mu)=-\frac{\bar{f}(0)}{\kappa_{2}(0)\left(\kappa_{2}(0)-\kappa_{1}(0)\right)}+O(\mu), \\
h_{3} T_{i}(t, \mu)=\left[\bar{\phi}_{i}\right]+O(\mu), i=1,2, h_{3} T_{3}(t, \mu)=1+\left[\bar{\phi}_{3}\right]+O(\mu), h_{3} Q(t, \mu)=[\bar{f}]+O(\mu),
\end{gathered}
$$

where

$$
\begin{gathered}
{\left[\bar{\phi}_{i}\right]=\int_{0}^{1} \frac{\bar{\phi}_{i}(s)}{y_{30}(s) A_{1}(s)}\left(y_{30}^{\prime}(1)-\int_{s}^{1} \sum_{j=0}^{2} a_{j}(x) y_{30}^{(j)}(x)-a_{1}(s) y_{30}(s)\right) d s-} \\
-\frac{a_{2}(0) \bar{\phi}_{i}(0)}{\kappa_{1}(0)\left(\kappa_{2}(0)-\kappa_{1}(0)\right)}+\frac{\bar{\phi}_{i}(1)}{\kappa_{1}(1)\left(\kappa_{2}(1)-\kappa_{1}(1)\right)}-\frac{a_{2}(1) \bar{\phi}_{i}(1)}{\kappa_{2}(1)\left(\kappa_{2}(1)-\kappa_{1}(1)\right)}, \quad i=\overline{1,3} \\
{[\bar{f}]=\int_{0}^{1} \frac{\bar{f}(s)}{y_{30}(s) A_{1}(s)}\left(y_{30}^{\prime}(1)-\int_{s}^{1} \sum_{j=0}^{2} a_{j}(x) y_{30}^{(j)}(x)-a_{1}(s) y_{30}(s)\right) d s-} \\
-\frac{a_{2}(0) \bar{f}(0)}{\kappa_{1}(0)\left(\kappa_{2}(0)-\kappa_{1}(0)\right)}+\frac{a_{2}(1) \bar{f}(1)}{\kappa_{1}(1)\left(\kappa_{2}(1)-\kappa_{1}(1)\right)}-\frac{\bar{f}(1)}{\kappa_{2}(1)\left(\kappa_{2}(1)-\kappa_{1}(1)\right)} .
\end{gathered}
$$

Then for the main determinant of the system (22):

$$
I(\mu)=\left|\begin{array}{lll}
h_{1} T_{1}(t, \mu) & h_{1} T_{2}(t, \mu) & h_{1} T_{3}(t, \mu) \\
h_{2} T_{1}(t, \mu) & h_{2} T_{2}(t, \mu) & h_{2} T_{3}(t, \mu) \\
h_{3} T_{1}(t, \mu) & h_{3} T_{2}(t, \mu) & h_{3} T_{3}(t, \mu)
\end{array}\right|
$$

we get the following asymptotic representation:

$$
I(\mu)=\left(1+\left[\bar{\phi}_{3}\right]\right)\left(1-\frac{\bar{\phi}_{2}(0)}{\kappa_{2}(0)\left(\kappa_{2}(0)-\kappa_{1}(0)\right)}\right)+\frac{\bar{\phi}_{3}(0)\left[\bar{\phi}_{2}\right]}{\kappa_{2}(0)\left(\kappa_{2}(0)-\kappa_{1}(0)\right)} \equiv \bar{I}+O(\mu) .
$$

C5) $\bar{I} \neq 0$.

Then from the system (22), we determine the unknown constants $C_{i}, i=1,2,3$. Thus, the following theorem will be true.

Theorem 1 If conditions (C1)-(C5) are satisfied, then the boundary value problem (1),(2) on the interval $[0,1]$ has a unique solution, expressed by formula $(16)$, where $T_{i}(t, \mu), i=$ $1,2,3, Q(t, \mu)$ are expressed by formula (17) and the constants $C_{i}, i=1,2,3$ are determined from system (22). 
For $C_{i}, i=1,2,3$ are valid asymptotic representations:

$$
\begin{gathered}
C_{1}=\alpha+O(\mu) ; C_{2}=\frac{1}{\bar{I}}\left[\alpha \cdot \frac{\bar{\phi}_{1}(0)\left(1+\left[\bar{\phi}_{3}\right]\right)-\bar{\phi}_{3}(0)\left[\bar{\phi}_{1}\right]}{\kappa_{2}(0)\left(\kappa_{2}(0)-\kappa_{1}(0)\right)}+\right. \\
\left.+\left(\beta+\frac{\bar{f}(0)}{\kappa_{2}(0)\left(\kappa_{2}(0)-\kappa_{1}(0)\right)}\right)\left(1+\left[\bar{\phi}_{3}\right]\right)+(\gamma-[\bar{f}]) \frac{\bar{\phi}_{3}(0)}{\kappa_{2}(0)\left(\kappa_{2}(0)-\kappa_{1}(0)\right)}\right]+O(\mu) ; \\
C_{3}=\frac{1}{\bar{I}}\left[\alpha\left(\frac{\bar{\phi}_{2}(0)\left[\bar{\phi}_{1}\right]-\bar{\phi}_{1}(0)\left[\bar{\phi}_{2}\right]}{\kappa_{2}(0)\left(\kappa_{2}(0)-\kappa_{1}(0)\right)}-\left[\bar{\phi}_{1}\right]\right)-\left(\beta+\frac{\bar{f}(0)}{\kappa_{2}(0)\left(\kappa_{2}(0)-\kappa_{1}(0)\right)}\right)\left[\bar{\phi}_{2}\right]+\right. \\
\left.+(\gamma-[\bar{f}])\left(1-\frac{\bar{\phi}_{2}(0)}{\kappa_{2}(0)\left(\kappa_{2}(0)-\kappa_{1}(0)\right)}\right)\right]+O(\mu) .
\end{gathered}
$$

Theorem 2 If conditions (C1)-(C5) are satisfied, then for solutions of problem (1),(2) are valid the following asymptotic representations:

$$
\begin{aligned}
& y^{(q)}(t, \mu)=\alpha\left[y_{30}^{(q)}(t)+\int_{0}^{t} \frac{y_{30}^{(q)}(t) \bar{\phi}_{1}(s)}{y_{30}(s) A_{1}(s)} d s-\frac{\kappa_{2}^{q-2}(t)-\kappa_{1}^{q-2}(t)}{\mu^{q-1}\left(\kappa_{2}(t)-\kappa_{1}(t)\right)} \cdot \bar{\phi}_{1}(t)-\right. \\
& -\frac{y_{10}(t) \kappa_{1}^{q}(t)}{\mu^{q-1} \kappa_{1}(0)}\left(y_{30}^{\prime}(0)+\frac{\bar{\phi}_{1}(0)}{\kappa_{1}(0)\left(\kappa_{2}(0)-\kappa_{1}(0)\right)}\right) e^{\frac{1}{\mu} \int_{0}^{t} \kappa_{1}(x) d x}-\frac{y_{20}(t) \kappa_{2}^{q}(t)}{\mu^{q-1} y_{20}(1) \kappa_{2}(1)} . \\
& \left.\cdot\left(\frac{h_{3} y_{30}(t)-a_{2}(0) y_{30}^{\prime}(0)}{1-a_{2}(1)}-\frac{\bar{\phi}_{1}(1)}{\kappa_{2}(1)\left(\kappa_{2}(1)-\kappa_{1}(1)\right)}\right) e^{\frac{1}{\mu} \int_{1}^{t} \kappa_{2}(x) d x}\right]+ \\
& +\frac{1}{\bar{I}}\left[\alpha \frac{\bar{\phi}_{1}(0)\left(1+\left[\bar{\phi}_{3}\right]\right)-\left[\bar{\phi}_{1}\right] \bar{\phi}_{3}(0)}{\kappa_{2}(0)\left(\kappa_{2}(0)-\kappa_{1}(0)\right)}+\right. \\
& \left.+\left(\beta+\frac{\bar{f}(0)}{\kappa_{2}(0)\left(\kappa_{2}(0)-\kappa_{1}(0)\right)}\right)\left(1+\left[\bar{\phi}_{3}\right]\right)+\frac{(\gamma-[\bar{f}]) \bar{\phi}_{3}(0)}{\kappa_{2}(0)\left(\kappa_{2}(0)-\kappa_{1}(0)\right)}\right] . \\
& {\left[\int_{0}^{t} \frac{y_{30}^{(q)}(t) \bar{\phi}_{2}(s)}{y_{30}(s) A_{1}(s)} d s-\frac{\kappa_{2}^{q-2}(t)-\kappa_{1}^{q-2}(t)}{\varepsilon^{q-1}\left(\kappa_{2}(t)-\kappa_{1}(t)\right)} \cdot \bar{\phi}_{2}(t)-\right.} \\
& -\frac{y_{10}(t) \kappa_{1}^{q}(t)}{\mu^{q-1} \kappa_{1}(0)}\left(\frac{\bar{\phi}_{2}(0)}{\kappa_{1}(0)\left(\kappa_{2}(0)-\kappa_{1}(0)\right)}-1\right) e^{\frac{1}{\mu} \int_{0}^{t} \kappa_{1}(x) d x}+ \\
& \left.+\frac{y_{20}(t) \kappa_{2}^{q}(t)}{\mu^{q-1} y_{20}(1) \kappa_{2}(1)}\left(\frac{\bar{\phi}_{2}(1)}{\kappa_{2}(1)\left(\kappa_{2}(1)-\kappa_{1}(1)\right)}-\frac{a_{2}(0)}{1-a_{2}(1)}\right) e^{\frac{1}{\mu} \int_{1}^{t} \kappa_{2}(x) d x}\right]+ \\
& +\frac{1}{\bar{I}}\left[\alpha\left(\frac{\bar{\phi}_{2}(0)\left[\bar{\phi}_{1}\right]-\left[\bar{\phi}_{2}\right] \bar{\phi}_{1}(0)}{\kappa_{2}(0)\left(\kappa_{2}(0)-\kappa_{1}(0)\right)}-\left[\bar{\phi}_{1}\right]\right)-\left(\beta+\frac{\bar{f}(0)}{\kappa_{2}(0)\left(\kappa_{2}(0)-\kappa_{1}(0)\right)}\right)\left[\bar{\phi}_{2}\right]+\right.
\end{aligned}
$$




$$
\begin{aligned}
& \left.+(\gamma-[\bar{f}])\left(1-\frac{\bar{\phi}_{2}(0)}{\kappa_{2}(0)\left(\kappa_{2}(0)-\kappa_{1}(0)\right)}\right)\right] \cdot\left[\int_{0}^{t} \frac{y_{30}^{(q)}(t) \bar{\phi}_{3}(s)}{y_{30}(s) A_{1}(s)} d s-\right. \\
& -\frac{\kappa_{2}^{q-2}(t)-\kappa_{1}^{q-2}(t)}{\mu^{q-1}\left(\kappa_{2}(t)-\kappa_{1}(t)\right)} \bar{\phi}_{3}(t)-\frac{\kappa_{1}^{q}(t) y_{10}(t) \bar{\phi}_{3}(0)}{\mu^{q-1} \kappa_{1}^{2}(0)\left(\kappa_{2}(0)-\kappa_{1}(0)\right)} e^{\frac{1}{\mu} \int_{0}^{t} \kappa_{1}(x) d x}+ \\
& \left.+\frac{y_{20}(t) \kappa_{2}^{q}(t)}{\mu^{q-1} y_{20}(1) \kappa_{2}(1)}\left(\frac{\bar{\phi}_{3}(1)}{\kappa_{2}(1)\left(\kappa_{2}(1)-\kappa_{1}(1)\right)}+\frac{1}{1-a_{2}(1)}\right) e^{\frac{1}{\mu} \int_{1}^{t} \kappa_{2}(x) d x}\right]+ \\
& +\int_{0}^{t} \frac{y_{30}^{(q)}(t) \bar{f}(s)}{y_{30}(s) A_{1}(s)} d s-\frac{\kappa_{1}^{q}(t) y_{10}(t) \bar{f}(0)}{\mu^{q-1} \kappa_{1}^{2}(0)\left(\kappa_{2}(0)-\kappa_{1}(0)\right)} e^{\frac{1}{\mu} \int_{0}^{t} \kappa_{1}(x) d x}+ \\
& +\frac{\kappa_{2}^{q}(t) y_{20}(t) \bar{f}(1)}{\mu^{q-1} \kappa_{2}^{2}(1) y_{20}(1)\left(\kappa_{2}(1)-\kappa_{1}(1)\right)} e^{\frac{1}{\mu} \int_{1}^{t} \kappa_{2}(x) d x}+\frac{\kappa_{1}^{q-2}(t)-\kappa_{2}^{q-2}(t)}{\mu^{q-1}\left(\kappa_{2}(t)-\kappa_{1}(t)\right)} \cdot \bar{f}(t)+ \\
& +O\left(\mu+\frac{1}{\mu^{q-2}} e^{\frac{1}{\mu} \int_{0}^{t} \kappa_{1}(x) d x}+\frac{1}{\mu^{q-2}} e^{\frac{1}{\mu} \int_{1}^{t} \kappa_{2}(x) d x}\right) \\
& +
\end{aligned}
$$

The proof of the theorem follows from (16) taking account of (21), (24).

From formula (25) for solutions of problem (1), (2), we obtain the following order of growth:

$$
y^{\prime \prime}(0, \mu)=O\left(\frac{1}{\mu}\right), \quad y^{\prime \prime}(1, \mu)=O\left(\frac{1}{\mu}\right), \mu \rightarrow 0 .
$$

It implies that at points $t=0$ and $t=1$ the solution of the considered problem (1), (2) have the first order boundary jumps.

\section{Modified degenerate problem}

Singularly perturbed boundary value problem (1), (2), we put in accordance with the following modified degenerate problem:

$$
\begin{gathered}
L_{0} \bar{y} \equiv A_{1}(t) \bar{y}^{\prime}(t)+A_{2}(t) \bar{y}(t)=F(t)+\int_{0}^{1} \sum_{i=0}^{2} H_{i}(t, x) \bar{y}^{(i)}(x) d x+\Theta(t), \\
h_{1} \bar{y} \equiv \bar{y}(0)=\alpha, \quad h_{2} \bar{y} \equiv \bar{y}^{\prime}(0)=\beta+\Theta_{0}, \\
h_{3} \bar{y} \equiv \bar{y}^{\prime}(1)-\int_{0}^{1} \sum_{i=0}^{2} a_{i}(x) \bar{y}^{(i)}(x) d x=\gamma+a_{2}(0) \Theta_{0}+\left(1-a_{2}(1)\right) \Theta_{1},
\end{gathered}
$$

where $\Theta(t)$ and $\Theta_{0}, \Theta_{1}$ are called jumps of the integral term and solution respectively. 
For the difference $u(t, \mu)$ between the solution $y(t, \mu)$ of the singularly perturbed problem (1), (2) and the solution $\bar{y}(t)$ of the degenerate problem, we get the problem:

$$
\begin{aligned}
& L_{\mu} u \equiv \mu^{2} u^{\prime \prime \prime}+\mu A_{0}(t) u^{\prime \prime}+A_{1}(t) u^{\prime}+A_{2}(t) u=\int_{0}^{1} \sum_{i=0}^{2} H_{i}(t, x) u^{(i)}(x, \mu) d x-\Theta(t)- \\
& -\mu^{2} y^{\prime \prime \prime}-\mu A_{0}(t) y^{\prime \prime}, \\
& h_{1} u \equiv u(0, \mu)=0, h_{2} u \equiv u^{\prime}(0, \mu)=-\Theta_{0}, \\
& h_{3} u \equiv u^{\prime}(1, \mu)-\int_{0}^{1} \sum_{i=0}^{2} a_{i}(x) u^{(i)}(x, \mu) d x=-a_{2}(0) \Theta_{0}-\left(1-a_{2}(1)\right) \Theta_{1} .
\end{aligned}
$$

The problem (28),(29) is of the same type as the problem (1),(2), by applying asymptotic formula (25). As a result, we obtain asymptotic representation for the solution of the problem (28), (29):

$$
\begin{aligned}
& u^{(q)}(t, \mu)=\frac{1}{\bar{I}}\left[-\left(\Theta_{0}+\frac{\bar{\Theta}(0)}{\kappa_{2}(0)\left(\kappa_{2}(0)-\kappa_{1}(0)\right)}\right)\left(1+\left[\bar{\phi}_{3}\right]\right)+\right. \\
& \left.-\frac{\left(a_{2}(0) \Theta_{0}+\left(1-a_{2}(1)\right) \Theta_{1}-[\bar{\Theta}]\right) \bar{\phi}_{3}(0)}{\kappa_{2}(0)\left(\kappa_{2}(0)-\kappa_{1}(0)\right)}\right] \cdot\left[\int_{0}^{t} \frac{y_{30}^{(q)}(t) \bar{\phi}_{2}(s)}{y_{30}(s) A_{1}(s)} d s-\frac{\kappa_{2}^{q-2}(t)-\kappa_{1}^{q-2}(t)}{\mu^{q-1}\left(\kappa_{2}(t)-\kappa_{1}(t)\right)} \bar{\phi}_{2}(t)-\right. \\
& -\frac{\kappa_{1}^{q}(t) y_{10}(t)}{\mu^{q-1} \kappa_{1}(0)}\left(\frac{\bar{\phi}_{2}(0)}{\kappa_{1}(0)\left(\kappa_{2}(0)-\kappa_{1}(0)\right)}-1\right) e^{\frac{1}{\mu} \int_{0}^{t} \kappa_{1}(x) d x}+ \\
& \left.+\frac{y_{20}(t) \kappa_{2}^{q}(t)}{\mu^{q-1} y_{20}(1) \kappa_{2}(1)}\left(\frac{\bar{\phi}_{2}(1)}{\kappa_{2}(1)\left(\kappa_{2}(1)-\kappa_{1}(1)\right)}-\frac{a_{2}(0)}{1-a_{2}(1)}\right) e^{\frac{1}{\mu} \int_{1}^{t} \kappa_{2}(x) d x}\right]+ \\
& +\frac{1}{\bar{I}}\left[\left(\Theta_{0}+\frac{\bar{\Theta}(0)}{\kappa_{2}(0)\left(\kappa_{2}(0)-\kappa_{1}(0)\right)}\right)\left[\bar{\phi}_{2}\right]+\right. \\
& \left.-\left(a_{2}(0) \Theta_{0}+\left(1-a_{2}(1)\right) \Theta_{1}-[\bar{\Theta}]\right)\left(1-\frac{\bar{\phi}_{2}(0)}{\kappa_{2}(0)\left(\kappa_{2}(0)-\kappa_{1}(0)\right)}\right)\right] \cdot\left[\int_{0}^{t} \frac{y_{30}^{(q)}(t) \bar{\phi}_{3}(s)}{y_{30}(s) A_{1}(s)} d s-\right. \\
& -\frac{\kappa_{2}^{q-2}(t)-\kappa_{1}^{q-2}(t)}{\mu^{q-1}\left(\kappa_{2}(t)-\kappa_{1}(t)\right)} \bar{\phi}_{3}(t)-\frac{\kappa_{1}^{q}(t) y_{10}(t) \bar{\phi}_{3}(0)}{\mu^{q-1} \kappa_{1}^{2}(0)\left(\kappa_{2}(0)-\kappa_{1}(0)\right.} e^{\frac{1}{\mu} \int_{0}^{t} \kappa_{1}(x) d x}+ \\
& \left.+\frac{y_{20}(t) \kappa_{2}^{q}(t)}{\mu^{q-1} y_{20}(1) \kappa_{2}(1)}\left(\frac{\bar{\phi}_{3}(1)}{\kappa_{2}(1)\left(\kappa_{2}(1)-\kappa_{1}(1)\right)}+\frac{1}{1-a_{2}(1)}\right) e^{\frac{1}{\mu} \int_{1}^{t} \kappa_{2}(x) d x}\right]-
\end{aligned}
$$




$$
\begin{gathered}
-\int_{0}^{t} \frac{y_{30}^{(q)}(t) \bar{\Theta}(s)}{y_{30}(s) A_{1}(s)} d s+\frac{\kappa_{1}^{q}(t) y_{10}(t) \bar{\Theta}(0)}{\mu^{q-1} \kappa_{1}^{2}(0)\left(\kappa_{2}(0)-\kappa_{1}(0)\right)} e^{\frac{1}{\mu} \int_{0}^{t} \kappa_{1}(x) d x}+ \\
-\frac{\kappa_{2}^{q}(t) y_{20}(t) \bar{\Theta}(1)}{\mu^{q-1} \kappa_{2}^{2}(1) y_{20}(1)\left(\kappa_{2}(1)-\kappa_{1}(1)\right)} e^{\frac{1}{\mu} \int_{1}^{t} \kappa_{2}(x) d x}+\frac{\kappa_{2}^{q-2}(t)-\kappa_{1}^{q-2}(t)}{\mu^{q-1}\left(\kappa_{2}(t)-\kappa_{1}(t)\right)} \bar{\Theta}(t)+ \\
+O\left(\mu+\frac{1}{\mu^{q-2}} e^{-\gamma_{1} \frac{t}{\mu}}+\frac{1}{\mu^{q-2}} e^{-\gamma_{2} \frac{1-t}{\mu}}\right), \quad q=\overline{0,2},
\end{gathered}
$$

where

$$
\bar{\Theta}(t) \equiv \Theta(t)+\int_{0}^{1} R_{0}(t, s, 1) \Theta(s) d s .
$$

If the function $\bar{\Theta}(t)$ is selected in the form

$$
\bar{\Theta}(t)=-\Theta_{0}\left(\bar{\phi}_{2}(t)+a_{2}(0) \bar{\phi}_{3}(t)\right)-\Theta_{1}\left(1-a_{2}(1)\right) \bar{\phi}_{3}(t),
$$

it can be presented that $u(t, \mu) \rightarrow 0, \mu \rightarrow 0$. From (32) in view of (18),(20), (31) to determine the jump of integral terms $\Theta(t)$, we obtain the following formula:

$$
\Theta(t)=\Theta_{0} H_{2}(t, 0)-\Theta_{1} H_{2}(t, 1) .
$$

Theorem 3 If conditions (C1)-(C5) are satisfied, then for the solution $y(t, \mu)$ of singularly perturbed integral boundary value problem (1),(2) are valid the limiting equalities:

$$
\begin{gathered}
\lim _{\mu \rightarrow 0} y(t, \mu)=\bar{y}(t), \quad 0 \leq t \leq 1, \\
\lim _{\mu \rightarrow 0} y^{(i)}(t, \mu)=\bar{y}^{(i)}(t), \quad i=1,2, \quad 0<t<1,
\end{gathered}
$$

where $\bar{y}(t)$ is a solution of modified degenerate boundary value problem (26),(27), the jump of the integral terms $\Theta(t)$ is determined by formula (33).

Example. To illustrate the results, we give an example. For simplicity, we consider the integral boundary value problem for a singularly perturbed integro-differential equation with constant coefficients:

$$
\begin{aligned}
& L_{\mu} y \equiv \mu^{2} y^{\prime \prime \prime}-\mu y^{\prime \prime}-2 y^{\prime}=1+\int_{0}^{1} \delta y^{\prime \prime}(x, \mu) d x, \quad \delta \neq 0, \\
& h_{1} y \equiv y(0, \mu)=\alpha, \quad h_{2} y \equiv y^{\prime}(0, \mu)=\beta, \quad h_{3} y \equiv y^{\prime}(1, \mu)-\int_{0}^{1} \nu y^{\prime \prime}(x, \mu) d x=\gamma, \quad \nu \neq 0, \nu \neq 1,
\end{aligned}
$$

where

$$
A_{0}(t)=-1, A_{1}(t)=-2, A_{2}(t)=0, F(t)=1, H_{i}(t, x)=0, i=0,1
$$




$$
H_{2}(t, x)=\delta, a_{i}(x)=0, i=0,1, a_{2}(x)=\nu .
$$

The exact solution to this problem is:

$$
\begin{gathered}
y(t, \mu)=\mu \cdot \frac{\delta(\gamma-\beta)+(2 \beta+1)(1-\nu)+[\beta(\delta+2 \nu)-\gamma(\delta+2)+\nu-1] e^{-\frac{2}{\mu}}}{2(1-\nu)\left(e^{-\frac{3}{\mu}}-1\right)} \cdot e^{-\frac{t}{\mu}}+ \\
+\mu \cdot \frac{\beta(\delta+2 \nu)-\gamma(\delta+2)+\nu-1+[\delta(\gamma-\beta)+(2 \beta+1)(1-\nu)] e^{-\frac{1}{\mu}}}{4(1-\nu)\left(e^{-\frac{3}{\mu}}-1\right)} \cdot e^{-\frac{2(t-1)}{\mu}}+\alpha- \\
-\mu \cdot \frac{2[\delta(\gamma-\beta)+(2 \beta+1)(1-\nu)]+3[\beta(\delta+2 \nu)-\gamma(\delta+2)+\nu-1] e^{-\frac{2}{\mu}}}{4(1-\nu)\left(e^{-\frac{3}{\mu}}-1\right)}- \\
-\mu \cdot \frac{[\delta(\gamma-\beta)+(2 \beta+1)(1-\nu)] e^{-\frac{3}{\mu}}}{4(1-\nu)\left(e^{-\frac{3}{\mu}}-1\right)}+\frac{\delta(\beta-\gamma)}{2(1-\nu)} \cdot t-\frac{t}{2} .
\end{gathered}
$$

Hence, passing to the limit at $\mu \rightarrow 0$, we get

$$
\lim _{\mu \rightarrow 0} y(t, \mu)=\alpha+\frac{\delta(\beta-\gamma)+\nu-1}{2(1-\nu)} t \equiv \bar{y}(t), \quad 0 \leq t \leq 1,
$$

where $\bar{y}(t)$ is not a solution to the usual degenerate equation, obtained from (1) at $\mu=0$, and is a solution of modified degenerate boundary value problem:

$$
\begin{aligned}
-2 \bar{y}^{\prime} & =1+\int_{0}^{1} \delta \bar{y}^{\prime \prime}(x) d x+\Theta(t), \\
h_{1} \bar{y} & \equiv \bar{y}(0)=\alpha, h_{2} \bar{y} \equiv \bar{y}^{\prime}(0)=\beta+\Theta_{0}, \\
h_{3} \bar{y} & \equiv \bar{y}^{\prime}(1)-\int_{0}^{1} \nu \bar{y}^{\prime \prime}(x) d x=\gamma+\nu \Theta_{0}+(1-\nu) \Theta_{1},
\end{aligned}
$$

where $\Theta_{0}, \Theta_{1}$ are jumps of the solution at points $t=0$ and $t=1$ respectively, determined from problem $(4.11),(4.12)$ and the value of the jump of the integral terms $\Delta(t)$ in accordance with formula (4.8) should be determined in the form:

$$
\Theta(t)=\delta\left(\Theta_{0}-\Theta_{1}\right)
$$

The exact solution of the problem (36), (37) according to the condition (38) has the form

$$
\bar{y}(t)=\alpha+\frac{\delta(\beta-\gamma)+\nu-1}{2(1-\nu)} t .
$$

Therefore, the passage to the limit (35) will be valid. The limit transitions are similarly valid.

$$
\lim _{\mu \rightarrow 0} y^{(i)}(t, \mu)=\bar{y}^{(i)}(t), \quad i=1,2, \quad 0<t<1 .
$$




\section{Conclusion}

In this paper, we study the boundary value problem for singularly perturbed integrodifferential equations with the phenomena of the so-called boundary jumps, when the fast solution variable becomes unbounded at both boundaries. The exceptions of the qualitative influence of integral terms on the asymptotic behavior of the solutions for singularly perturbed integro-differential equations are shown. The presence of integral terms will significantly change the degenerate equation: the solution of the assumed singularly perturbed integrodifferential equation does not tend to the solution of the usual degenerate equation, obtained from the supposed equation with the zero value of a small parameter and will tend to solve a specially modified degenerate integro-differential equation with an additional term called the jump of the integral term. Moreover, the value of the jump of the integral term is determined differently than in [33] because of occurence of jumps in solutions of the same order. In addition, in the boundary conditions of the modified degenerate problem, there is also a change. The so-called jumps of the first derivative of the solution appear $\Theta_{0}=\bar{y}^{\prime}(0)-y^{\prime}(0, \mu) \neq 0$ and $\Theta_{1}=\bar{y}^{\prime}(1)-y^{\prime}(1, \mu) \neq 0$ at the ends of considered interval.

\section{References}

[1] Schlesinger L. "Uber asymptotische darstellungen der losungen linearer differential systeme als funktionen eines parameters", Mathematische Annalen, (1907); 63(3): 277-300.

[2] Birkhoff GD. "On the asymptotic character of the solutions of certain linear differential equations containing a parameter" , Transactions of the American Mathematical Society, (1908); 9(2): 219-231.

[3] Noaillon P. "Developpements asymptotiques dans les equations differentielles lineaires a parametre variable", Mem. Soc. Sci. Liege, (1912); 3(11): 197.

[4] Wasow W. "Singular perturbations of boundary value problems for nonlinear differential equations of the second order", Comm. On Pure and Appl. Math., (1956); 9 : 93-113.

[5] Nayfeh A. "Perturbation Methods", New York, USA: John Wiley, (1973).

[6] Tihonov AN. "O zavisimosti reshenij differencial'nyh uravnenij ot malogo parametra", Matematicheskij sbornik (1948); 22(2): 193-204 (in Russian).

[7] Tihonov AN. "O sistemah differencial'nyh uravnenij soderzhashhih parametry", Matematicheskij sbornik, (1950); 27(69): $147-156$ (in Russian).

[8] Vishik MI, Lyusternik LA. "Regular degeneration and boundary layer for linear differential equations with small parameter multiplying the highest derivatives", Usp.Mat. Nauk, (1957); 12: 3-122 (in Russian), Amer. Math. Soc. Transl. 1962; 20(2): $239-364$.

[9] Vishik MI, Lyusternik LA. "On the initial jump for non-linear differential equations containing a small parameter" ,Doklady Akademii Nauk SSSR, (1960); 132(6): 1242-1245 (in Russian).

[10] Bogoliubov N, Mitropolskii YA. "Asymptotic methods in the theory of nonlinear oscillations",Delhi: Hindustan Publ. Corp., (1961).

[11] Vasil'eva A, Butuzov V. "Singularly perturbed differential equations of parabolic type in Asymptotic Analysis II" , Lecture Notes in Math. Berlin: Springer-Verlag, (1983).

[12] Vasil'eva A, Butuzov V, Kalachev L. "The boundary function method for singular perturbation problems" ,Philadeplhia: SIAM Studiesin Applied Mathematics, (1995).

[13] O’Malley R. "Introduction to singular perturbations" ,New York, USA: Academic Press, (1974).

[14] O’Malley R. "Singular perturbations methods for ordinary differential equations", Berlin: Springer-Verlag, (1991). 
[15] Smith DR. "Singular-perturbation theory", Cambridge: Cambridge University Press, (1985).

[16] Eckhaus W. "Matched asymptotic expansions and singular perturbations", London: North-Holland, (1973).

[17] Chang K, Howes F. "Nonlinear Singular Perturbation Phenomena: Theory and Application",New York, USA: Springer Verlag, (1984).

[18] Kevorkian J, Cole JD. "Singular perturbation methods in applied mathematics", Berlin: Springer-Verlag, (1981).

[19] Sanders, Verhulst F. "Averaging methods in nonlinear dynamical systems", Berlin: Springer-Verlag, (1985).

[20] Mishchenko E, Rozov N. "Differential equations with small parameter and relaxation oscillations" , New York, USA: Plenum Press, (1980).

[21] Lomov S. "Introduction to the general theory of singular perturbations", American Mathematical Society, Providence, (1992).

[22] Imanaliev MI. "Asimptoticheskie metody v teorii singulyarno vozmushchennyh integro-differencial'nyh sistem" , Frunze: Ilim, (1972) (in Russian).

[23] Kassymov KA. Ob asimptotike reshenija zadachi Koshi s bol'shimi nachal'nymi uslovijami dlja nelinejnogo obyknovennogo differencial'nogo uravnenija, soderzhashhego malyj parametr, Uspehi matematicheskih nauk, (1962); 17(5): 187-188 (in Russian).

[24] Kassymov KA. "O zadache s nachal'nym skachkom dlja nelinejnyh sistem differencial'nyh uravnenij soderzhashhih malyj parametr" , DAN SSSR (1968); 179(2): 275-278 (in Russian).

[25] Kassymov KA. "Asimptotika reshenija zadachi s nachal'nymi skachkami dlja sistemy differencial'nyh uravnenij giperbolicheskogo tipa s malym parametrom pri proizvodnoj", DAN SSSR, (1971); 196(2): 274-277 (in Russian).

[26] Vishik MI, Lyusternik LA. "O nachal'nom skachke dlya nelinejnyh differencial'nyh uravnenij, soderzhashchih malyj parametr", DAN SSSR, (1960); 132(6): 1242-1245 (in Russian).

[27] Kasymov KA. "Ob asimptotike resheniya zadachi Koshi s bol'shimi nachal'nymi usloviyami dlya nelinejnogo obyknovennogo differencial'nogo uravneniya, soderzhashchego malyj parametr", UMN, (1962); 17(5): 187-188 (in Russian).

[28] Kassymov KA, Nurgabyl D. "Asymptotic estimates of solution of a singularly perturbed boundary value problem with an initial jump for linear differential equations", Differential Equations (2004); 40(5): 641-651.

[29] Dauylbayev MK, Atakhan N. "The initial jumps of solutions and integral terms in singular BVP of linear higher order integro-differential equations", Miskolc Math. Notes (2015); 16(2): 747-761.

[30] Kassymov KA, Dauylbaev MK. "Estimation of solutions to problems associated with linear singularly perturbed integrodifferential equations", Differential Equations, (1999); 35(6): 822-830.

[31] Dauylbaev MK. "The asymptotic behavior of solutions to singularly perturbed nonlinear integro-differential equations" ,Siberian Mathematical Journal, (2000); 41(1): 49-60.

[32] Dauylbaev MK, Mirzakulova AE. "Boundary value problems with initial jumps for singularly perturbed integrodifferential equations" , Journal of Mathematical Sciences, (2017); 222(3): 214-225. doi: 10.1007/s10958-017-3294-7

[33] Dauylbaev MK, Mirzakulova AE. "Asymptotic behavior of solutions of singular integro-differential equations", Journal of Discontinuity, Nonlinearity, and Complexity, (2016); 5(2): 147-154.

[34] Akhmet M, Dauylbayev M, Mirzakulova A. "A singularly perturbed differential equation with piecewise constant argument of generalized type" , Turkish Journal of Mathematics, (2018); 42(4): 1680-1685.

[35] Dauylbayev, M.K., Uaissov, A.B. "Asymptotic Behavior of the Solutions of Boundary-Value Problems for Singularly Perturbed Integrodifferential Equations", Ukrainian Mathematical Journal, (2020); 71(11): 1677-1691 